\title{
Intoxicação aguda com sementes de Crotalaria spectabilis (Leg. Papilionoideae) em suínos ${ }^{1}$
}

\author{
Daniel G. Ubiali², Fabiana M. Boabaid ${ }^{3}$, Nayani A. Borges ${ }^{4}$, Flávio H.B. Caldeira², \\ Luciano R. Lodi², Caroline A. Pescador², Marcos A. Souza² e Edson M. Colodel ${ }^{2 *}$
}

\begin{abstract}
Ubiali D.G., Boabaid F.M., Borges N.A., Caldeira F.H.B., Lodi L.R., Pescador C.A., Souza M.A. \& Colodel E.M. 2011. [Acute poisoning with Crotalaria spectabilis (Leg. Papilionoideae) seeds in pigs.] Intoxicação aguda com sementes de Crotalaria spectabilis (Leg. Papilionoideae) em suínos. Pesquisa Veterinária Brasileira 31(4):313318. Departamento de Clínica Médica Veterinária, Faculdade de Agronomia e Medicina Veterinária e Zootecnia, Universidade Federal de Mato Grosso, Av. Fernando Corrêa da Costa 2367, Bairro Coxipó, Cuiabá, MT 78068-900, Brazil. E-mail: moleta@ufmt.br

Hepatocellular necrosis are reported in swine after consumption of diets containing grains of "sorgo-granífero" (Sorghum bicolor) accidentally contaminated with Crotalaria spectabilis seeds in the municipality of Juscimeira, MT. Clinical signs began 24-48 hours after consumption of contaminated ration and were characterized by depression, lethargy, apathy, loss of appetite, vomiting, pale or jaundiced mucous membranes, ascites, lateral recumbency, the lateral position with paddling and convulsions, clinical outcome was 4860 hours followed by death. 76 pigs died in four properties. The main gross lesions were liver increased in size and lobular illustration with red-brown central areas interspersed with yellowish areas, ascites and hydrothorax with reddish-yellow liquid containing filaments with aspect of fibrin, enlarged lymph nodes and interlobular pulmonary edema. The disease was experimentally reproduced with 16 pigs divided into six groups that received seeds of C. spectabilis in different doses. Hepatocellular necrosis occurred in seven pigs, two of which received daily doses of $2.5 \mathrm{~g} / \mathrm{kg}$ and five who received single doses of 5 and $9.5 \mathrm{~g} /$ $\mathrm{kg}$. Ten daily doses of 0.5 and $1.25 \mathrm{~g} / \mathrm{kg}$ caused liver fibrosis.
\end{abstract}

INDEX TERMS: Poisonous plants, Crotalaria spectabilis, Leg. Papilionoideae, pyrrolizidine alkaloids, plant poisoning, acute hepatic necrosis, swine diseases, pathology.

RESUMO.- Relata-se necrose hepatocelular em suínos após consumo de ração que continha grãos de sorgo-granífero (Sorghum bicolor) acidentalmente contaminado com sementes de Crotalaria spectabilis. Morreram 76 suínos em quatro propriedades no município de Juscimeira, MT. Os sinais clínicos iniciaram-se 24-48 horas após o consumo da ração contaminada e foram caracterizados por depressão, letargia,

\footnotetext{
${ }^{1}$ Recebido em 8 de setembro de 2010.

Aceito para publicação em 17 de novembro de 2010.

2 Departamento de Clínica Médica Veterinária, Faculdade de Agronomia, Medicina Veterinária e Zootecnia, Universidade Federal de Mato Grosso (UFMT), Av. Fernando Corrêa da Costa 2367, Bairro Coxipó, Cuiabá, MT 78068-900, Brasil. *Autor para correspondência: moleta@ufmt.br

${ }^{3}$ Setor de Patologia Veterinária, Universidade Federal do Rio Grande do Sul (UFRGS), Av. Bento Gonçalves 9090, Porto Alegre, RS 91540-000, Brasil.

${ }^{4}$ Farmacêutica Autônoma, Rua Oriente Tenuta 268, Apto 603, Bairro Consil, Cuiabá, MT 78048-490.
}

apatia, inapetência, vômito, mucosas ictéricas ou pálidas, ascite, decúbito esternal, decúbito lateral com movimentos de pedalagem e convulsões, a evolução clínica foi de 48-60 horas seguida de morte. As Principais alterações macroscópicas foram fígado aumentado de tamanho com evidenciação do padrão lobular, ascite e hidrotórax com líquido de coloração amarelo avermelhado contendo filamentos com aspecto de fibrina, linfonodos aumentados e edema pulmonar interlobular. A doença foi reproduzida utilizando-se 16 suínos divididos em seis grupos que receberam sementes de $C$. spectabilis em diferentes doses. Necrose hepatocelular ocorreu em sete suínos, sendo dois que receberam doses diárias $2,5 \mathrm{~g} / \mathrm{kg}$ e cinco que receberam doses únicas de 5,0 e 9,5g/kg. Dez doses diárias de 0,5 e 1,25g/kg causaram fibrose hepática.

TERMOS DE INDEXAÇÃO: Plantas tóxicas, Crotalaria spectabilis, Leg. Papilionoideae, alcalóides pirrolizidínicos, intoxicação por planta, necrose hepática aguda, doenças de suínos, patologia. 


\section{INTRODUÇÃO}

Plantas do gênero Crotalaria são conhecidas popularmente no Brasil por "chocalho", "guizo-de-cascavel", "chocalho-de-cascavel", "chocalho-de-cobra", "feijão-de-guizo" ou "xique-xique (Boghossian et al. 2007, Riet-Correa et al. 2007). Crotalaria spectabilis Roth, da família Fabaceae, é utilizada em sistemas de rotação de cultura, adubação verde, cobertura morta, fixação de nitrogênio atmosférico e controle de nematóides na agricultura (Souza et al. 1997, Erasmo et al. 2004, Silveira \& Rava 2004).

Algumas espécies do gênero Crotalaria são tóxicas para animais domésticos por conter alcalóides pirrolizidínicos (APs), dessas Crotalaria spectabilis é provavelmente a espécie mais tóxica (Neal et al. 1935, Tokarnia \& Döbereiner 1982).

A intoxicação por $C$. spectabilis tem sido observada em diversas espécies de animais domésticos sendo os suínos os mais sensíveis, seguido pelos frangos, bovinos, eqüinos, ovinos e caprinos (Torres et al. 1997, Tokarnia et al. 2000).

As plantas desse gênero podem causar intoxicação aguda ou crônica, sendo esta última mais freqüente. No Brasil foram descritos surtos espontâneos de intoxicação crônica por $C$. juncea em equinos e $C$. mucronata em bovinos em Minas Gerais (Nobre et al. 1994, Lemos et al. 1997) e C. retusa em eqüinos, bovinos e ovinos na Paraíba (Nobre et al. 2004, Riet-Correa et al. 2009). Experimentalmente foram reproduzidas intoxicações crônicas com C. spectabilis em suínos (Souza et al. 1997, Torres et al. 1997), C. mucronata e C. anagyroides em bovinos (Tokarnia \& Döbereiner 1982, 1983, Boghossian et al. 2007). As intoxicações agudas foram descritas em ovinos que ingeriram grandes quantidades de C. retusa (Nobre at al. 2005).

Nas criações intensivas de suínos as condições de criação e de manejo diminuem as possibilidades de acontecer intoxicações por plantas. Estas podem ocorrer quando sementes ou outras partes tóxicas são acidentalmente misturadas a grãos e/ou fazendo parte da formulação da ração para os animais (Timm \& Riet-Correa 1997, Galey 2006).

Relata-se o quadro epidemiológico, clínico e patológico da intoxicação acidental e experimental por sementes de Crotalaria spectabilis em suínos.

\section{MATERIAL E MÉTODOS}

Intoxicação acidental. No mês de agosto de 2008 ocorreu mortalidade em suínos em quatro propriedades (A, B, C e D) no município de Juscimeira, MT, Brasil, após a utilização de uma partida de sementes de sorgo de uma fábrica de ração do mesmo município. A equipe do Laboratório de Patologia Veterinária da Universidade Federal de Mato Grosso (LPV-UFMT) visitou as propriedades, fábrica de ração e a unidade produtora de sorgo no município de Santo Antônio do Leverger, MT. O histórico clínico e os dados epidemiológicos foram coletados com o médico veterinário responsável pela fábrica de ração e com os proprietários dos criatórios. Foram necropsiados cinco suínos na Propriedade A e um suíno na Propriedade B, sendo coletadas amostras de vísceras conservadas em formol a $10 \%$ para exame microscópico de rotina.

Amostras de sorgo e ração foram coletadas na fábrica, nas propriedades onde ocorreram os surtos e na unidade produto-
Quadro 1. Delineamento da intoxicação experimental com sementes de Crotalaria spectabilis em suínos

\begin{tabular}{|c|c|c|c|c|c|c|}
\hline $\begin{array}{c}\text { Grupo/ } \\
\text { Suíno }\end{array}$ & $\begin{array}{c}\text { Peso } \\
(\mathrm{kg})\end{array}$ & $\begin{array}{l}\text { Dose } \\
(\mathrm{g} / \mathrm{kg})\end{array}$ & $\begin{array}{c}\mathrm{N}^{\circ} \\
\text { Doses }\end{array}$ & $\begin{array}{l}\text { Início } \\
\text { dos sinais } \\
\text { clínicos }\end{array}$ & $\begin{array}{c}\text { Desfecho/ } \\
\text { Evolução }\end{array}$ & $\begin{array}{l}\text { Princi- } \\
\text { pal lesão }\end{array}$ \\
\hline \multirow[t]{3}{*}{1} & 9,2 & 0,5 & 10 & $108 \mathrm{~h}$ & $E^{a / 10 d}$ & Fibrose \\
\hline & 9,2 & & 10 & $122 \mathrm{~h}$ & $E / 10 d$ & \\
\hline & 8,2 & & 10 & $147 \mathrm{~h}$ & $E / 20 d$ & \\
\hline \multirow[t]{3}{*}{2} & 12 & 1,25 & 10 & $93 \mathrm{~h}$ & $E / 10 d$ & Fibrose \\
\hline & 6,1 & & 10 & $123 \mathrm{~h}$ & $E / 10 d$ & \\
\hline & 7,2 & & 10 & $127 \mathrm{~h}$ & $E / 20 d$ & \\
\hline \multirow[t]{2}{*}{3} & 8,7 & 2,5 & 8 & $72 \mathrm{~h}$ & $M / 8 d$ & Necrose \\
\hline & 9 & & 5 & $120 \mathrm{~h}$ & $M / 6 d$ & \\
\hline \multirow{3}{*}{4} & 8,5 & 2,5 & 1 & - & $E / 20 d$ & - \\
\hline & 8,2 & & 1 & - & $E / 20 d$ & \\
\hline & 5 & & 1 & - & $E / 20 d$ & \\
\hline \multirow{4}{*}{5} & 5,7 & 5 & 1 & $48 \mathrm{~h}$ & $M / 3 d$ & Necrose \\
\hline & 8,2 & & 1 & $4 \mathrm{~h}$ & $M / 24 h$ & \\
\hline & 10 & & 1 & $6 \mathrm{~h}$ & $\mathrm{M} / 24 \mathrm{~h}$ & \\
\hline & 11 & & 1 & $50 \mathrm{~h}$ & $M / 3 d$ & \\
\hline 616 & 6,4 & 9,5 & 1 & $3 \mathrm{~h}$ & $\mathrm{M} / 24 \mathrm{~h}$ & Necrose \\
\hline
\end{tabular}

a $\mathrm{E}=$ Eutanasiado, $\mathrm{M}=$ morte, $\mathrm{d}=$ dias, $\mathrm{h}=$ horas.

ra de sorgo granífero. O nível de contaminação das amostras de sorgo com Crotalaria spectabilis foi estipulada pela separação manual das sementes de Crotalaria spectabilis em amostras de grãos de sorgo das diferentes origens. Amostras botânicas de $C$. spectabilis foram coletadas na unidade produtora de sorgo e encaminhadas para o Herbário Central da UFMT para classificação morfológica.

Intoxicação experimental. Utilizaram-se 16 suínos, clinicamente sadios, mestiços, nove fêmeas e sete machos, pesando entre cinco e $12 \mathrm{~kg}$, adquiridos junto ao Setor de Suinocultura da Fazenda Experimental da UFMT (SSFE-UFMT), foram divididos em seis grupos apresentados no Quadro 1. Estes suínos foram mantidos no recinto experimental do LPVUFMT, em baias, recebendo ração para fase de crescimento, obtida no SSFE-UFMT e água fornecidas ad libitum. As sementes de $C$. spectabilis foram moídas e administradas, por via oral, com auxilio de uma seringa de $20 \mathrm{ml}$ com o cilindro cortado na parte anterior. Os suínos foram acompanhados clinicamente durante o período experimental e avaliados quanto ao apetite, disposição em movimentar-se, coloração das mucosas, aspecto das fezes e da urina e estado corporal.

Estudo microscópico. Durante a necropsia dos suínos acidental e experimentalmente intoxicados coletou-se fragmentos de fígado, pulmão, sistema nervoso central, rim, coração, baço, linfonodos, tonsilas, esôfago, estômago e intestino, fixou-se em solução de formalina a 10\%. Em seguida as amostras foram rotineiramente processadas para análise histológica, corados pela Hematoxilina \& Eosina e pelo Tricrômico de Masson (Prophet et al. 1992).

\section{Intoxicação acidental}

\section{RESULTADOS}

Foram visitadas as Propriedades A, B e D que tinham criação extensiva de suínos e utilizavam ração comercial formulada com $25 \%$ de sorgo contaminado com sementes de Crotalaria spectabilis e a Propriedade B que utilizava sorgo contaminado triturado adquirido na mesma fábrica de ração, o qual foi misturado com casquinha de soja numa proporção de $50 \%$. 
Na Propriedade A, eram criados 96 suínos. A ração contaminada foi disponibilizada nos cochos por um período de 12 horas para consumo ad libitum. Aproximadamente 36 horas após o fornecimento da ração, 28 suínos foram encontrados mortos e nove apresentaram sinais clínicos de depressão, letargia, inapetência e morte, após evolução clínica entre 24 e 30 horas. Por recomendação do médico veterinário toda a ração foi recolhida após constatação das mortes. Foram necropsiados cinco suinos, quatro dos quais encontrados mortos e um eutanasiado in extremis.

As principais alterações macroscópicas foram observadas no fígado que estava aumentado de tamanho (Fig.2A), com evidenciação do padrão lobular na superfície capsular e de corte (Fig.2B), caracterizada por áreas vermelho-escuro circundadas por discretas áreas vermelho-claras, havia líquido de coloração amarelo-avermelhado contendo grumos com aspecto de fibrina nas cavidades abdominal, torácica e pericárdica. Os linfonodos estavam aumentados, notou-se edema pulmonar interlobular e edema de mesocólon. Histologicamente a principal lesão foi caracterizada como necrose hepatocelular coagulativa centrolobular a massiva (Fig.2C,D).

$\mathrm{Na}$ Propriedade B, os suínos eram cruzados com Javali (Sus scrofa), o rebanho era composto por 60 animais, destes, cinco adoeceram e morreram. O inicio dos sinais clínicos variou de 24 a 48 horas após a disponibilização da ração. Os suídeos apresentaram depressão, anorexia, letargia, decúbito esternal com movimentos de pedalagem, convulsões e morte com evolução em torno de 30 horas. Durante a visita um suídeo apresentava sinais clínicos e morreu durante o exame sendo imediatamente necropsiado. Os achados macro e microscópicos foram semelhantes aos encontrados na Propriedade A. Relatou-se adicionalmente que 20 galinhas, dentre um lote de 100 criadas soltas nesta propriedade, morreram após receberem o sorgo contaminado.

Segundo relato do médico veterinário da fábrica de ração na Propriedade $\mathrm{C}$ morreram 12 suínos, após 12 horas da disponibilização da ração comercial, com quadro clínico e patológico similar às Propriedades A e B. Esse veterinário reco- mendou o recolhimento da ração e sorgo contaminados nas Propriedades A, B e C armazenando na Propriedade D, pertencente ao proprietário da fábrica de ração. Essa ração e sorgo contaminados foram acidentalmente fornecidos a 50 suínos, sendo que 22 morreram com quadro clínico similar ao descrito nas outras propriedades.

Estimou-se uma concentração de aproximadamente 2\% de sementes de C. spectabilis misturados aos grãos de sorgo (Fig.1B). Na ração utilizada nas Propriedades A, C e D estimou-se uma contaminação de $0,5 \%$ e na ração da Propriedade B estimou-se 1\%. Constatou-se que a contaminação do sorgo ocorreu acidentalmente durante o beneficiamento das sementes na unidade produtora de grãos, $C$. spectabilis era usada como adubo verde em sistema de rotação em culturas de sorgo e soja. As amostras botânicas foram classificadas como Crotalaria spectabilis Roth (Fabaceae) (Fig.1A).

\section{Intoxicação experimental}

Nos Grupos 1 e 2, cada um com três suínos, receberam respectivamente, 0,5 e $1,25 \mathrm{~g} / \mathrm{kg}$ durante 10 dias. Os sinais clínicos iniciaram em média 120 horas após a primeira dose de sementes $C$. spectabilis administrada, foram caracterizados por icterícia, fraqueza, andar cambaleante, má condição corporal e pêlos arrepiados. Em cada grupo dois suínos foram sacrificados aos 10 e o outro aos 20 dias após a primeira dose administrada. Na necropsia notou-se diminuição do volume do fígado, que normalmente apresentava de $70-80 \%$ do volume normal, e ocasionalmente estava com metade do tamanho normal. Os lobos hepáticos estavam fusionados devido à deposição de substância com aspecto de fibrina, a consistência estava aumentada e a cápsula de Glisson espessada. A coloração na superfície capsular e de corte variava de amareloalaranjado com áreas irregulares marrom-claras e vermeIho-escuras. Adicionalmente, observou-se hidrotórax, ascite, edema nas serosas intestinais, edema pulmonar interlobular e hemorragias petequiais e equimóticas em diferentes órgãos. As alterações histológicas (Quadro 2),

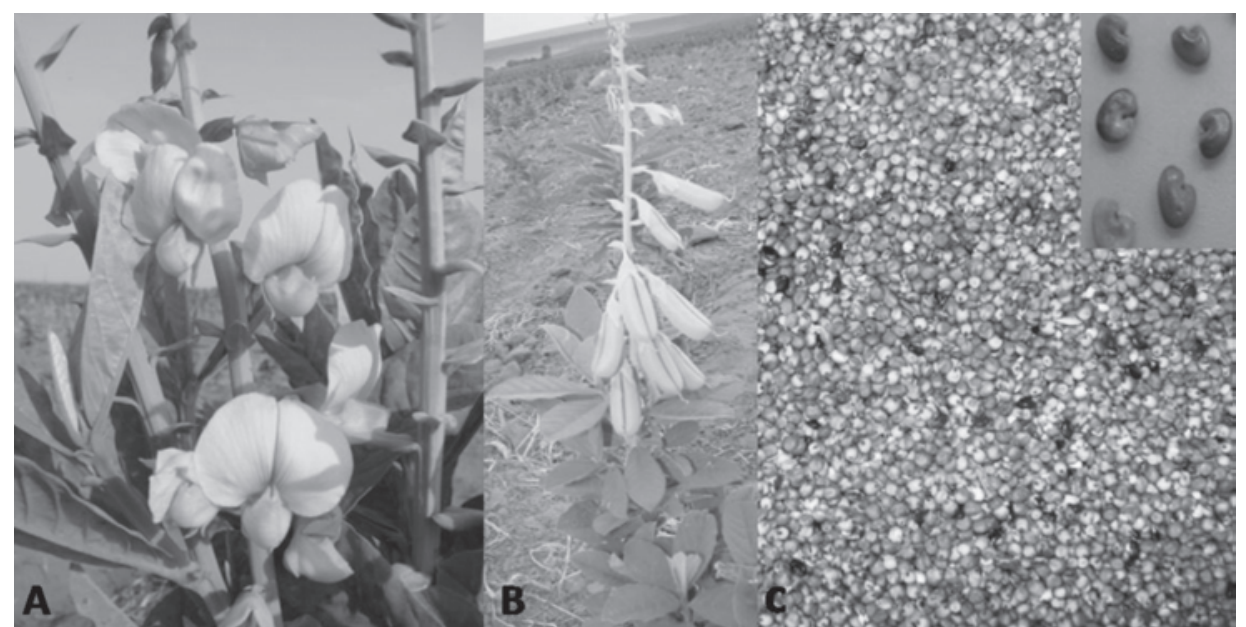

Fig.1. Crotalaria spectabilis, em agosto de 2008, Santo Antônio de Leverger, MT. (A) Inflorescência. (B) Frutificação. (C) Grãos de Sorghum bicolor contaminados com sementes de $C$. spectabilis (detalhe). 


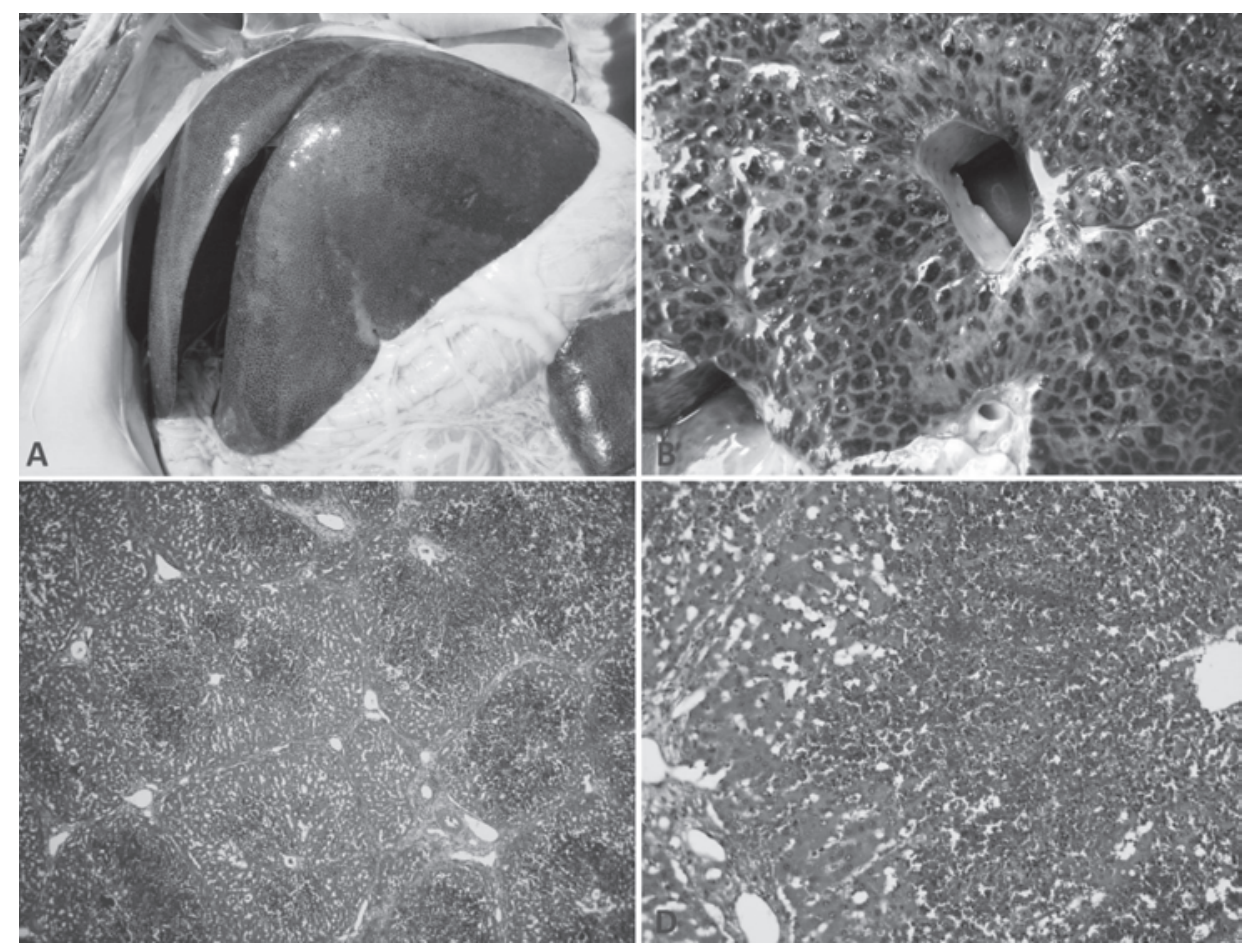

Fig.2. Intoxicação acidental por Crotalaria spectabilis em suínos. (A) Fígado aumentado em volume e com evidenciação do padrão lobular na superfície capsular. (B) Na superfície de corte. (C) Microscopicamente caracterizado por necrose hepatocelular massiva, congestão e hemorragia. HE, obj.10x. (D) Magnificação da imagem anterior. HE, obj.40x.

Quadro 2. Principais alterações histológicas no fígado de suínos experimentalmente intoxicados por Crotalaria spectabis

\begin{tabular}{|c|c|c|c|c|c|c|c|c|}
\hline \multicolumn{2}{|c|}{$\begin{array}{l}\text { Grupo/ } \\
\text { Suíno }\end{array}$} & \multirow{2}{*}{$\begin{array}{c}\text { Necrose } \\
-a\end{array}$} & \multirow{2}{*}{$\begin{array}{c}\text { Hemorragia } \\
++\end{array}$} & \multirow{2}{*}{$\begin{array}{c}\text { Fibrose } \\
-\end{array}$} & \multirow{2}{*}{$\begin{array}{c}\text { Hiperplasia de } \\
\text { ductos biliares }\end{array}$} & \multirow{2}{*}{$\begin{array}{c}\text { Megalocitose } \\
+\end{array}$} & \multirow{2}{*}{$\begin{array}{c}\text { Diminuição de } \\
\text { lóbulos } \\
++\end{array}$} & \multirow{2}{*}{$\begin{array}{c}\begin{array}{c}\text { Necrose } \\
\text { individual } \\
\text { hepatócito }\end{array} \\
++\end{array}$} \\
\hline 1 & 1 & & & & & & & \\
\hline & 2 & - & - & - & - & + & ++ & ++ \\
\hline & 3 & - & - & $\mathrm{IL}+{ }^{\mathrm{C}}$ & + & + & + & + \\
\hline \multirow[t]{3}{*}{2} & 4 & - & - & - & - & - & + & + \\
\hline & 5 & - & - & IL+ & - & + & + & + \\
\hline & 6 & - & - & IL, IT+ & + & ++ & +++ & + \\
\hline \multirow[t]{2}{*}{3} & 7 & $M^{b}$ & +++ & - & - & - & - & - \\
\hline & 8 & $M$ & +++ & - & - & + & - & - \\
\hline \multirow[t]{3}{*}{4} & 9 & - & - & - & - & - & - & - \\
\hline & 10 & - & - & - & - & - & - & - \\
\hline & 11 & - & - & - & - & - & - & - \\
\hline \multirow[t]{4}{*}{5} & 12 & C & ++ & - & - & + & - & - \\
\hline & 13 & C & ++ & - & - & - & - & - \\
\hline & 14 & $M$ & +++ & - & - & - & - & - \\
\hline & 15 & $M$ & +++ & - & - & + & - & - \\
\hline 6 & 16 & $M$ & +++ & - & - & - & - & - \\
\hline
\end{tabular}

a Padrão de intensidade de lesões: +++ acentuada, ++ moderada, + leve, - ausente. ${ }^{\text {b }}$ Padrão de localização de necrose: $\mathrm{M}=$ massiva, $\mathrm{C}=$ centrolobular. $^{\mathrm{C}}$ Padrão de localização de fibrose: $\mathrm{IL}=$ interlobular, $\mathrm{IT}=$ intralobular.

nos Grupos 1 e 2 foram diminuição acentuada no tamanho dos lóbulos hepáticos, relacionada à redução do número e volume de hepatócitos, necrose hepatocelular individual e leve desorganização de cordões celulares. Em dois suínos do Grupo 2 e um do Grupo 1 observou-se fibrose predominantemente perilobular, e por vezes entre cordões de hepatócitos, nestes havia megalocitose hepatocelular ocasional, colestase e leve proliferação de ductos biliares.
O Grupo 3 recebeu 2,5g/kg durante cinco e oito dias, sendo que o ínicio dos sinais clínicos foi de 72 a $120 \mathrm{~h}$ e a evolução clínica foi de 144 a 192 h. O Grupo 5 recebeu $5 \mathrm{~g} /$ $\mathrm{kg}$ em dose única, sendo que o inicio dos sinais clínicos variou de 4 a 50 h e a evolução clínica de 24 a 72 h. O Grupo 6 recebeu 9,5g/kg o início dos sinais foi de $3 \mathrm{~h}$ e a evolução foi de $24 \mathrm{~h}$. Os sinais clínicos foram semelhantes nos três grupos e caracterizados por apatia, anorexia, au- 


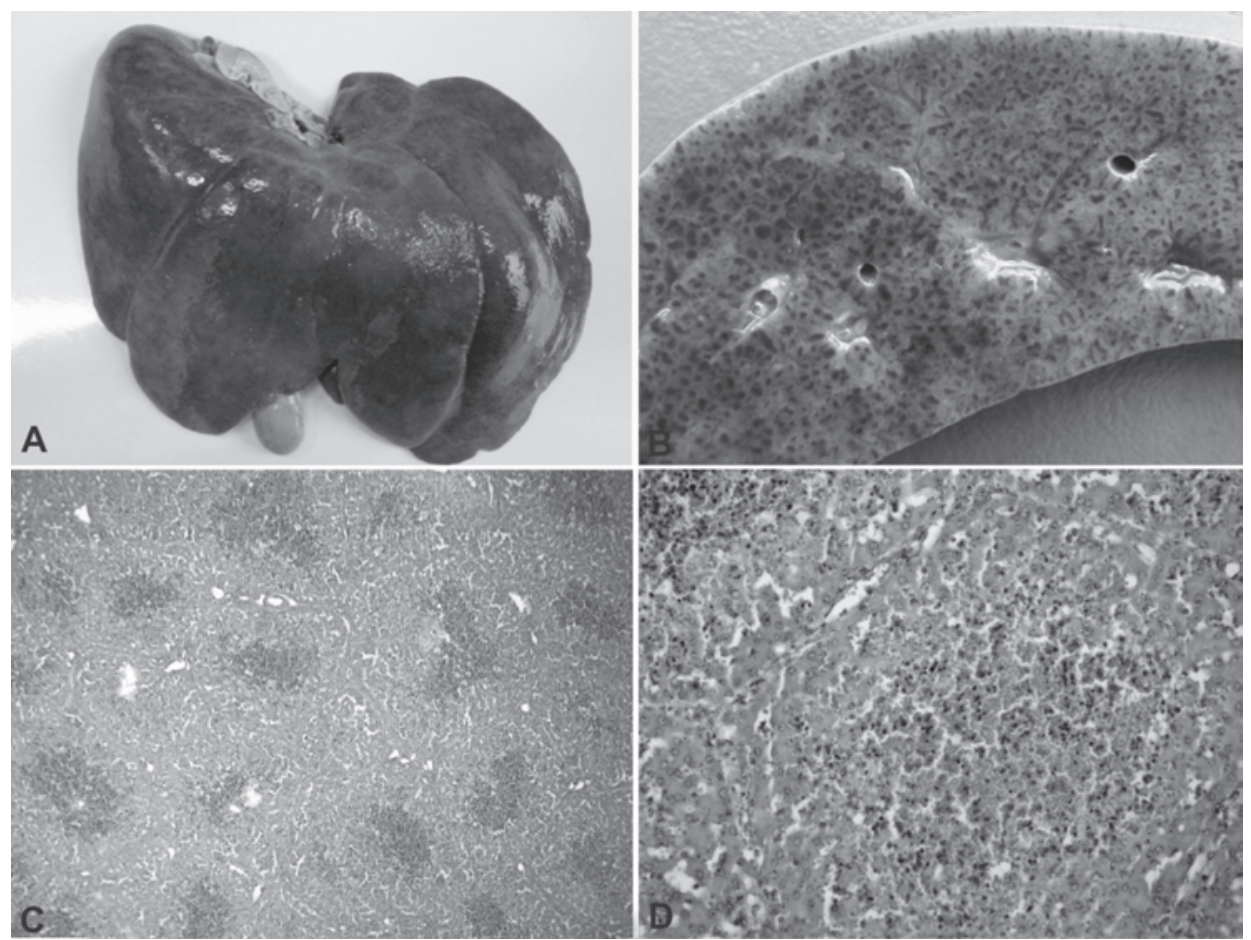

Fig.3. Intoxicação experimental por Crotalaria spectabilis, por dose única de 5,0g/kg. (A) Figado aumentado em volume. (B) Superfície de corte do fígado com acentuação do padrão lobular. (C) Necrose hepatocelular massiva, congestão e hemorragia. HE, obj.5x. (D) Magnificação da imagem anterior. HE, obj.40x.

mento do volume abdominal, redução da sensibilidade a estímulos externos, tremores, convulsões, depressão, decúbito lateral e marcada dispnéia momentos antes da morte. Nos suínos do Grupo 3 havia icterícia moderada e nos dos Grupos 5 e 6 leve. No Grupo 5 dois suínos tiveram vômito, epistaxe e melena três dias após a última dose.

$\mathrm{Na}$ necropsia dos suínos dos Grupos 3, 5 e 6 observou-se aumento do volume hepático (Fig.3A), evidenciação do padrão lobular com áreas vermelho-escuras centrais entremeadas por faixas claras na superfície natural e de corte (Fig.3B). Havia icterícia, líquido amarelo-avermelhado em grande quantidade nas cavidades abdominal e torácica, linfonodos aumentados, hemorragias petequiais e/ou equimóticas no rim, epicárdio, mucosa duodenal e tecido subcutâneo. No suíno que recebeu oito doses de $2,5 \mathrm{~g} / \mathrm{kg}$ houve sufusão estendendo-se nas serosas gástrica e duodenal. Histologicamente as principais alterações foram no fígado, caracterizadas por necrose hepatocelular centrolobular a massiva associada a hemorragia e congestão (Fig.3C,D). Nesses fígados com lesões agudas houve ocasionalmente megalocitose, colestase, dilatação de sinusóides hepáticos e do espaço de Disse, esferóides eosinofílicos intracitoplasmáticos, vacuolização hepatocelular e desorganização dos cordões de hepatócitos. Nesses três Grupos havia mielinopatia espongiforme (status spongiosus) principalmente na substância branca das folhas e substância basal do cerebelo, medula oblonga e mesencéfalo. Sendo mais evidente no suíno que apresentou evolução clínica de 192 horas do Grupo 3.
Três suínos do Grupo 4 receberam dose única de 2,5g/ $\mathrm{kg}$, foram eutanasiados após um período de 20 dias, sem apresentar sinais clínicos e alterações morfológicas.

\section{DISCUSSÃO}

No Brasil são descritas as formas aguda e crônica da intoxicação por APs. As plantas envolvidas nesses casos pertencem aos gêneros Senecio, Crotalaria e Echium (RietCorrea et al. 2009, Lucena et al. 2010). A fibrose hepática decorrente do consumo de C. spectabilis é experimentalmente descrita por Torres et al.(1997) e por Souza et al.(1997). Neste trabalho relatamos necrose hepatocelular associada ao consumo espontâneo de sementes de Crotalaria spectabilis em suínos. Esse quadro foi reproduzido experimentalmente através da administração de doses únicas a partir de $5 \mathrm{~g} / \mathrm{kg}$ ou doses diárias de $2,5 \mathrm{~g} / \mathrm{kg}$. Doses de $1,25 \mathrm{~g} / \mathrm{kg}$ e $0,5 \mathrm{~g} / \mathrm{kg}$ diariamente administradas causaram lesões iniciais de fibrose hepática.

O diagnóstico da intoxicação por sementes dessa planta foi baseado nos dados epidemiológicos, sinais clínicos, achados patológicos, observação de sementes misturadas aos grãos de sorgo utilizado nas rações e através da reprodução experimental do quadro clínico e patológico através da administração de sementes de $C$. spectabilis a suínos.

A toxidez do gênero Crotalaria está relacionada à concentração de APs, esta determina a evolução aguda ou crônica nos animais, isso foi observado em ovinos e caprinos que pastoreavam em áreas infestadas por $C$. retusa em fase de sementação, onde desenvolveram necrose 
hepatocelular (Lucena et al. 2010). Há bastante variação no teor de APs nas diferentes espécies de plantas ou em diferentes partes de uma mesma planta. Anjos et al. (2010) demonstram que a concentração de APs (alcalóide monocrotalina) nas sementes de $C$. retusa é de até 40 vezes maior que as outras partes da planta.

$\mathrm{Na}$ intoxicação acidental neste trabalho, as rações utilizadas nas Propriedades A e B continham entre 0,5 e 1\% de sementes de $C$. spectabilis, o que causou necrose hepática com evolução entre 24 e 48 horas. Baseado em dados sobre o consumo de ração e peso dos suínos (Barbosa et al. 1988), estimamos que os mesmos consumiram uma dose de aproximadamente $1 \mathrm{~g} / \mathrm{kg}$ de sementes de $C$. spectabilis. Experimentalmente observamos necrose hepática em suínos que receberam dose única acima de $5 \mathrm{~g} / \mathrm{kg}$, e doses repetidas de $2,5 \mathrm{~g} / \mathrm{kg}$ durante 5 e 8 dias. Torres et al. (1997) estudaram os efeitos da ingestão de ração contendo $1 \%$ de sementes de $C$. spectabilis para suínos e notaram necrose hepática em um animal que consumiu a mistura por 8 dias. Acreditamos que essa diferença, entre os resultados de literatura e os dados da reprodução experimental neste trabalho em relação ao caso natural, pode estar relacionada à linhagem genética, sistema de criação e/ou falhas de homogeneização na ração.

APs são compostos químicos estáveis e são bioativados no fígado pelas enzimas Citocromo P-450 para metabólicos tóxicos denominados ésteres e alcoóis pirrólicos. Esses derivados são agentes alquilantes altamente reativos que inibem a mitose, causando megalocitose e morte celular. (Lucena et al. 2010). Altas doses de APs causam necrose de hepatócitos principalmente na região centrolobular (Anjos et al. 2010).

Os suínos que receberam as doses repetidas de $0,5 \mathrm{e}$ $1,25 \mathrm{~g} / \mathrm{kg}$ durante 10 dias, foram eutanasiados imediatamente ou dez dias após a última dose. Nestes havia um quadro clínico de insuficiência hepática associada a diminuição do volume hepático e caracterizada microscopicamente por redução do tamanho lobular, necrose individual, megalocitose ocasional e fibroplasia discreta a leve. Torres et al. (1997) e Souza et al. (1997), verificaram fibrose hepática acentuada em suínos que receberam ração contaminada com $C$. spectabilis em doses entre 0,2 e $0,6 \%$ por períodos acima de 70 dias de consumo. A fibrose discreta observada neste estudo está relacionada provavelmente ao curto período de consumo de sementes de $C$. spectabilis.

Agradecimentos.- Ao Conselho Nacional de Desenvolvimento Científico e Tecnológico-CNPq, Intitutos Nacionais de Ciência e Tecnologia Proc.573534/2008-0, pelo suporte financeiro.

\section{REFERÊNCIAS}

Anjos B.L., Nobre V.M.T., Dantas A.F.M., Medeiros R.M.T., Neto T.S.O., Molyneux R.J. \& Riet-Correa F. 2010. Poisoning of sheep by seeds of Crotalaria retusa: Acquired resistance by continuous administration of low doses. Toxicon 55:28-32.

Barbosa H.P., Lima G.J.M.M. \& Ferreira A.S. 1988. Estimativa da quantidade de ração necessária para produção de um suíno com
100 kg de peso vivo. Comun. Téc. 133, Embrapa Suínos e Aves, Concórdia, SC.

Boghossian M.R., Peixoto P.V., Brito M.F. \& Tokarnia C.H. 2007. Aspectos clínico-patológicos da intoxicação experimental pelas sementes de Crotalaria mucronata (Fabaceae) em bovinos. Pesq. Vet. Bras. 27(4):149-156.

Erasmo E.A.L., Azevedo W.R., Sarmento R.A., Cunha A.M. \& Garcia S.L.R. 2004. Potencial de espécies utilizadas como adubo verde no manejo integrado de plantas daninhas. Planta Daninha 22:337342.

Galey F.D. 2006. Plantas e outras substâncias tóxicas naturais, p.801803. In: Smith B.P. (Ed.), Medicina Interna de Grandes Animais. $3^{\text {a }}$ ed. Manole, São Paulo.

Lemos R.A.A., Dutra I.S., Souza G.F., Nakazato L. \& Barros C.S.L. 1997. Intoxicação espontânea por Crotalaria mucronata em bovinos em Minas Gerais. Arqs Inst. Biológico, São Paulo, 64:46.

Lucena R.B., Rissi D.R., Maia L.A., Flores M.M., Dantas A.F.M., Nobre V.M.T., Riet-Correa F. \& Barros C.S.L. 2010. Intoxicação por alcaloides pirrolizidínicos em ruminantes e equinos no Brasil. Pesq. Vet. Bras. 30(5):447-452.

Neal W.M., Rusoff L.L. \& Ahmann C.F. 1935. The isolation and some properties of an alkaloid from Crotalaria spectabilis Roth. J. Am. Chem. Soc. 57(12):2560-2561.

Nobre D., Dagli M.L.Z. \& Haraguchi M. 1994. Crotalaria juncea intoxication in horses. Vet. Hum. Toxicol. 36(5):445-448.

Nobre V.M.T., Riet-Correa F., Barbosa Filho J.M., Dantas A.F.M., Tabosa I.M. \& Vasconcelos J.S. 2004. Intoxicação por Crotalaria retusa (Fabaceae) em eqüídeos no semi-árido da Paraíba. Pesq. Vet. Bras. 24(3):132-143.

Nobre V.M.T., Dantas A.F.M., Riet-Correa F., Barbosa Filho J.M., Tabosa I.M. \& Vasconcelos J.M. 2005. Acute intoxication by Crotalaria retusa in sheep. Toxicon 45:347-352.

Prophet E.B., Mills B., Arrington J.B. \& Sobin L.H. 1992. Laboratory Methods in Histotechnology. American Registry of Pathology, Armed Forces Institute of Pathology, Washington, DC. 279p.

Riet-Correa F. \& Méndez M.D.C. 2007. Intoxicações por plantas e micotoxinas: plantas hepatotóxicas, p.99-114. In: Riet-Correa F., Schild A.L., Lemos R.A.A. \& Borges J.R.J. (Eds), Doenças de Ruminantes e Eqüídeos. Vol.2. 3를 ed. Palotti, Santa Maria. 719p.

Riet-Correa F., Medeiros R.M.T., Pfister J., Schild A.L. \& Dantas A.F.M. 2009. Poisoning by Plants, Mycotoxins and Related Substances in Brazilian Livestock. Palotti, Patos. 246p.

Silveira P.M. \& Rava C.A. 2004. Utilização de crotalária no controle de nematóides da raiz do feijoeiro. Embrapa. Comun. Téc. 74, Santo Antônio de Goiás.

Souza A.C., Hatayde M.R. \& Bechara G.H. 1997. Aspectos patológicos da intoxicação de suínos por sementes de Crotalaria spectabilis Fabaceae. Pesq. Vet. Bras. 17(1):12-18.

Timm C.D. \& Riet-Correa F. 1997. Plantas tóxicas para suínos. Ciência Rural 27(3):521-528.

Tokarnia C.H. \& Döbereiner J. 1982. Intoxicação experimental por Crotalaria mucronata (Leg. Papilionoideae) em bovinos. Pesq. Vet. Bras. 2(2):77-85.

Tokarnia C.H. \& Döbereiner J. 1983. Intoxicação experimental por Crotalaria anagyroides (Leg. Papilionoideae) em bovinos. Pesq. Vet. Bras. 3(4):115-123.

Tokarnia C.H., Döbereiner J. \& Peixoto P.V. 2000. Plantas Tóxicas do Brasil. Editora Helianthus, Rio de Janeiro. 309p.

Torres M.B.A.M., Salles M.W.S., Headley A.S. \& Barros C.S.L. 1997. Intoxicação experimental por sementes de Crotalaria spectabilis Leguminosae em Suínos. Ciência Rural 27(2):307-312. 